\title{
Numerical simulation of influence of boundary slip on lubrication performances considering cavitation of textured surface
}

\author{
Quandai Wang ${ }^{+}$, Yulong Sun, Bingbing Guo, Xiaoli Hou, Pengyang Li and Yan Li \\ School of Mechanical and Precision Instrument Engineering, Xi' an University of Technology, Xi'an,710048
}

\begin{abstract}
A finite element calculation model of friction pairs with different micro texture features was established. Considering the condition of surface texture cavitation, numerical calculation was carried out by computational fluid dynamics (CFD) software to study the influence of the fluid-solid boundary slip on the lubrication performance of the friction pair under the hydrodynamic lubrication condition. The results demonstrate that the friction and bearing capacity of the lubricating film are reduced by the boundary slip, and the calculation results of the lubrication performance are influenced by the boundary condition of the cavitation caused by the micro texture; using the cavitation boundary condition, the boundary slip effect is enhanced and the theoretical comprehensive lubrication performance is improved; Compared with texture with cross section of triangle, rectangle and ellipse, the spherical texture facilitates carrying capacity improvement and there is the texture geometry size that facilitates lubrication property optimization.
\end{abstract}

Keywords: Numerical simulation, Lubrication performance, Cavitation, Boundary slip, Micro-texture

\section{Introduction}

The classical lubrication theory holds that there is no relative motion between the molecules of the lubricating medium and the solid surface, which has solved many problems of fluid lubrication ${ }^{[1]}$. However, it is impossible to withstand infinite shear stress between the liquid molecules or the solid-liquid interface molecules, therefore, in theory, the interaction between solid and liquid molecules can be overcome as long as the shear stress is high enough. With the help of modern micro-nano detecting technology and molecular dynamics simulation technology, it is shown that the assumption of non-slip at the liquid-solid interface in the classical fluid mechanics and lubrication theory does not always hold ${ }^{[2]}$. In fact, in many cases, the slip length can reach tens or even hundreds of microns ${ }^{[3]}$.

The insignificant boundary slip at macroscopic scale may cause significant changes in the friction and bearing capacity at the micro lubrication gap of the bearing. Spikes ${ }^{[4]}$ based on the finite difference method and the limit shear stress model, the effect of the boundary slip on the hydrodynamic behavior of the sliding block bearing is analyzed when only the surface slips occurs on the stationary sliding block. It is found that when the ultimate shear stress of the sliding surface is equal to zero, the hydrodynamic bearing capacity of the bearing is half that of the non-slip surface, but the corresponding surface friction force is reduced by several orders of magnitude. Considering the slip and applying the flow conservation boundary condition to the cavitation area, the classical Reynolds equation was modified and the parameters of the partial slip surface were optimized through simulation by Zhang Hui ${ }^{[5]}$. The boundary slip effect on the load capacity is closely related to the matching of the sliding property between the motion and the static surface. When the ultimate shear stress on the sleeve surface (static surface) is equal to zero, the bearing can still maintain a certain bearing capacity, while the ultimate shear stress on the journal surface (moving surface) is equal to zero or small enough, the bearing ultimately completely loses its bearing capacity ${ }^{[6-7]}$.

\footnotetext{
Corresponding author. Tel.: + 8602982312672 ; fax: +86 02982312670 .

E-mail address: quandaiw@163.com.
} 
The non-wetting surface facilitates larger boundary slippage formation. The phenomena of superhydrophobic surfaces provides a possible for the design and manufacture of surfaces that can easily lead to boundary slip ${ }^{[8-9]}$. In order to achieve superhydrophobic surface, surface microtexture is needed ${ }^{[10]}$. The experimental results show that the appropriate microstructure shape and distribution will produce the dynamic pressure effect and then improve load capacity ${ }^{[11-12]}$. The first explanation of the dynamic pressure effect of microstructures is given by Hamilton ${ }^{[13]}$. The dynamic pressure effect is considered to be the reason that a single protruding of the texture is equivalent to a small dynamic pressure axis. The influence of surface texture and partial slip on bearing capacity of sliding bearing is analyzed by Tauviqirrahman and the influence of texture area, depth width ratio and slip length of texture is given ${ }^{[14]}$. The micro texture causes cavitation in the lubrication flow field. When the surface texture effect on the lubrication property is studied, the cavitation effect should not be ignored ${ }^{[15]}$.

In order to study how the surface slip and texture affect the lubrication performance, a finite element calculation model of the friction pair with the different geometric features of the surface texture is established. Considering the cavitation effect of the surface texture, the numerical simulation is carried out using CFD software. The research results can be used for reference in surface texture design and lubrication calculation in drag reduction applications by interface slip.

\section{Theoretical model and numerical method}

\subsection{Computational model}

The single micro texture size is very small relative to the macroscopic friction pair and the effect of the surface curvature of the friction pair is ignored. The wall surface is simplified as a plane and the rotation speed is simplified as linear motion of the upper wall. The calculation model of various texture sections is shown as shown in figure 1. The parameters are as follows: $L$ is the length of the fluid field along the $\mathrm{x}$ direction, the $H_{0}$ is the thickness of the lubricant film, the $u$ is the relative velocity of the upper wall, the $w$ is the width of the micro texture, and the $d$ is the depth of the micro texture.

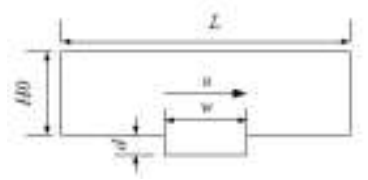

(a) rectangular

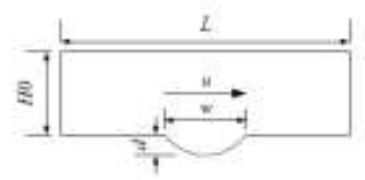

(b) spherical

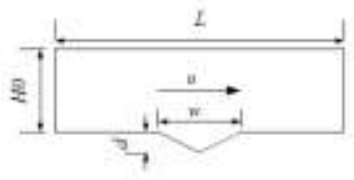

(c) triangular

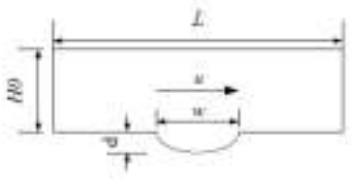

(d) elliptical

Fig.1 Computational model of textured surfaces with various cross-sectional geometric features: (a) rectangular; (b) spherical; (c) triangular; (d) elliptical.

\subsection{Governing equations}

\section{1) Governing equations for two phase flow considering cavitation effect}

Because of sudden change of the gap length caused by the micro texture, cavitation will occur in the lubrication field. Under this condition, the lubricating medium can be regarded as a mixture of two phases (gas phase and liquid phase). Therefore, the continuity equation and momentum equation of the lubricating fluid can be written as:

$$
\begin{gathered}
\frac{\partial}{\partial t}\left(\rho_{m}\right)+\nabla \cdot\left(\rho_{m} \overrightarrow{v_{m}}\right)=0 \\
\frac{\partial}{\partial t}\left(\rho_{m} \overrightarrow{v_{m}}\right)+\nabla \cdot\left(\rho_{m} \overrightarrow{v_{m}} \overrightarrow{v_{m}}\right)=-\nabla p+\nabla \cdot\left[\mu_{m}\left(\nabla \overrightarrow{v_{m}}+\nabla{\overrightarrow{v_{m}}}^{T}\right)\right]+\rho_{m} \vec{g}
\end{gathered}
$$

The mixture density of the lubricant is:

$$
\rho_{m}=\alpha_{v} \rho_{v}+\left(1-\alpha_{v}\right) \rho_{l}
$$

In order to close the equations, the mass transfer equation is needed to be solved:

$$
\frac{\partial}{\partial t}\left(\alpha_{v} \rho_{v}\right)+\nabla \cdot\left(\alpha_{v} \rho_{v} \overrightarrow{v_{m}}\right)=R_{e}-R_{c}
$$


where

$v_{m}$ - speed of mixed lubricants; $p$ - pressure of the lubricant film; $\mu_{m}-$ mixed dynamic viscosity of lubricants; $\rho_{v}$ - density of gas phase lubricants; $\rho_{l}$ - density of liquid lubricants; $\alpha_{v}$-volume fraction of the gas phase of the lubricant; $R_{e}, R_{c}$ - source term of mass transfer between gas and liquid phase.

\section{2) Cavitation model}

The effect of the cavitation effect caused by the sudden change of gap length is described by the SchnerrSauer cavitation model ${ }^{[16]}$. The saturated vapor pressure of the cavitation pressure is $p_{\mathrm{c}}=3540 \mathrm{~Pa}$ and the equation of the source phase $R_{\mathrm{e}}$ and $R_{c}$ is as follows:

$$
R_{e}=\frac{\rho_{v} \rho_{l}}{\rho_{m}} \alpha(1-\alpha) \frac{3}{R_{B}} \sqrt{\frac{2}{3} \frac{p_{c}-p}{\rho_{l}}}
$$

When $p_{\mathrm{c}}>p$

$$
R_{c}=\frac{\rho_{v} \rho_{l}}{\rho_{m}} \alpha(1-\alpha) \frac{3}{R_{B}} \sqrt{\frac{2}{3} \frac{p-p_{c}}{\rho_{l}}}
$$

When $p_{c}<p$, The radius $R_{\mathrm{B}}$ of the bubble is determined by the following formula:

$$
R_{B}=\left(\frac{\alpha}{1-\alpha} \frac{3}{4 \pi} \frac{1}{n_{0}}\right)^{1 / 3}
$$

$n_{0}$-The number density constant of the bubble and the default value of $n_{0}=10^{13}$ is adopted.

\section{3) Slip model}

According to the fluid lubrication mechanics, the limit shear stress model is as follows:

When $\tau<\tau_{\mathrm{c}}, \tau=\tau_{0}$,

When $\tau \geqslant \tau_{\mathrm{c}}, \tau=\tau_{\mathrm{c}}$,

Initial shear stress on the wall without slip is:

$$
\tau_{0}=\left(y-\frac{h}{2}\right) \frac{\partial p}{\partial x}+\frac{\mu}{h} u
$$

Where $\tau$ is the shear stress on the wall of the lubricating fluid, $\tau_{0}$ is the initial shear stress and $\tau_{c}$ is the ultimate shear stress on the wall; $\mu$ is the dynamic viscosity of the lubricating fluid; $h$ is the thickness of the lubricating film; $p$ is the lubricating film pressure and $x$ is the direction of movement of the lubricants, $y$ is perpendicular to $x$.

\subsection{Calculation of hydrodynamic lubrication properties}

By integrating the lubricant film pressure, the bearing capacity of lubricant between the parallel surfaces with micro textures can be obtained:

$$
F_{y}=\iint p d s
$$

The friction force between the parallel surfaces is obtained by integrating the viscous shear force on the upper surface:

$$
F_{x}=\iint \tau d s
$$

where $s$ is contact area; $\tau$ is shearing force. The parameters are nondimensionalized as followings:

$$
\begin{aligned}
& p^{+}=\frac{10^{-4} p H_{0}^{2}}{\eta u L} \quad w^{+}=\frac{w}{L}, d^{+}=\frac{d}{H_{0}}, \quad x^{+}=2 \frac{x}{L} \quad x \in\left\{-\frac{L}{2}, \frac{L}{2}\right\}, \\
& F_{x}^{+}=\frac{F_{x} H_{0}^{2}}{\eta u L} \quad F_{y}^{+}=\frac{F_{y} H_{0}^{2}}{\eta u L} .
\end{aligned}
$$




\subsection{Numerical method and boundary conditions}

In this paper, FLUENT software is used to calculate lubrication properties without considering the change of temperature. Liquid water is used as lubricating fluid and its dynamic viscosity is $1.003 \mathrm{mPa} . \mathrm{s}$.

The boundary conditions used in the calculation are as follows: the upper wall uses a no-slip boundary condition and moves along the $\mathrm{x}$ direction; The lower wall surface is fixed using the conditions of no slip or complete slip boundary; Periodic boundary conditions are used for the left and right boundaries to simulate regularly arranged micro texture arrays in fluid regions.

\section{Results and Discussion}

\subsection{Effect of boundary slip on lubrication performances}

A rectangular micro texture model is used to calculate the effect of wall slip on the fluid lubrication performance without considering the cavitation of the lubricating film. The pressure distribution of the flow field is shown in figure 2. Figure 3 is the pressure distribution curve on the upper wall.

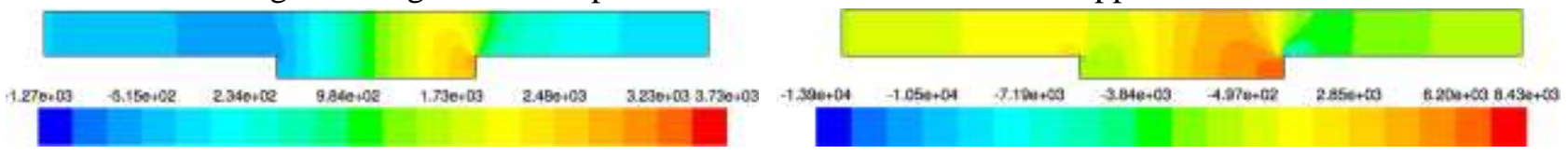

(a) no slip

(b) slip

Fig. 2: Pressure distribution under different wall slip conditions with $w^{+}=0.3$ and $d^{+}=0.5$

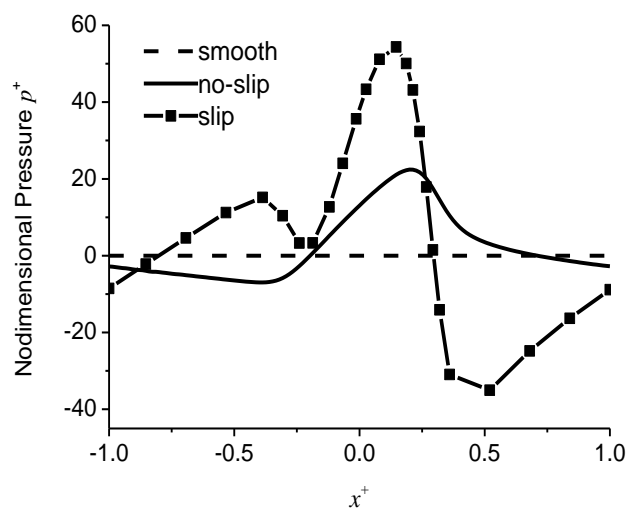

Fig. 3: Pressure distribution in the upper wall for different conditions with $w^{+}=0.3$ and $d^{+}=0.5$

From figure 2 it can be seen that the negative pressure is formed at the entrance of the micro texture due to the gap size expanding suddenly and positive pressure is formed at the exit of the micro texture due to the gap size shrinking suddenly. The dynamic pressure produced by the micro texture is not antisymmetry along the line $p^{+}=0$. The introduction of micro texture into the flow field produces additional positive pressure. It can be seen from the pressure curve that the pressure rises from the inlet of the flow field and drops at the entrance of the micro texture. Then the pressure begins to increase to the pressure peak at the outlet of the texture. Under slip boundary condition, the maximum value of the pressure is higher than that in the absence of slip. However, after passing the highest pressure point, the pressure drop on the sliding wall surface is larger than that of non slip condition. Under slip boundary, there is a large area of negative pressure in the back end of the flow field which counteracts the positive pressure and then on the whole, reduces the bearing capacity of the lubricating film, which is consistent with the research conclusions in the literature ${ }^{[17]}$.

\subsection{Effect of cavitation on lubrication performances}

In order to study the effect of the cavitation effect caused by the micro texture on the wall slip calculation results, the pressure distribution of the fluid field is calculated by taking the cavitation effect of the micro texture into consideration. Figure 4 is the volume fraction cloud pictures of the gas phase in the flow field when the cavitation effect is considered. The pressure distribution on the upper wall is shown in figure 5 . 


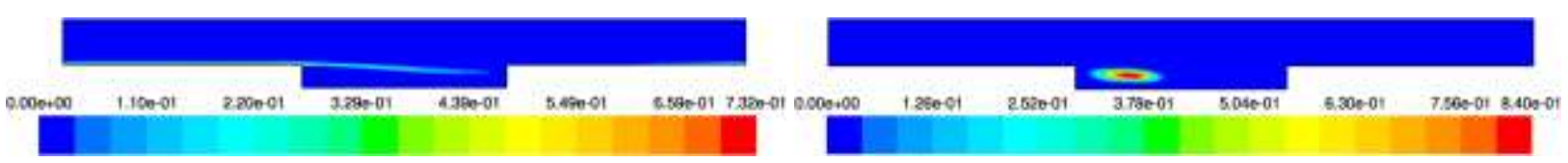

(a) no slip, cavitation

(b) slip, cavitation

Fig. 4: Contours plot of vapor volume fraction for different slip conditions considering cavitation at $w^{+}=0.3$ and $d^{+}=0.5$ :

(a) no-slip, (b) slip.

It can be seen from figure 4 that cavitation occurs in the diverging region of the micro texture, and the cavitation area is larger when no slip on the lower wall. When the wall is completely slipping, cavitation area is focused near the entrance of the flow field. From the pressure distribution curve of the upper wall, it can be seen that the maximum pressure value at the outlet of the micro texture is obviously greater than that in the absence of cavitation. At the same time, when the wall slip and cavitation effect are taken into account, the pressure in the inlet area of the flow field is obviously greater than that of only cavitation consideration and the peak pressure of the outlet end of the micro texture is two times more than that of no slip condition. This is because the increase of the flow rate at the inlet of the flow field makes the pressure of the lubricating film rise.

\subsection{Effect of the cross section shape of texture on lubrication performance}

The full slip boundary condition is used on the lower wall, and the cavitation effect of the lubricating film is considered. The effect of the shape of the micro texture section on the lubrication performance is studied by the calculation model as shown in figure 1 . The pressure distribution on the upper wall is shown in figure 6 , from which, we can see that the dynamic pressure formed by the spherical surface and the triangular micro texture is larger. Since from point of view of manufacturing, the micro texture with the microsphere shape is good for machining, in the subsequent calculation cases, the micro texture with spherical shape is adopted.

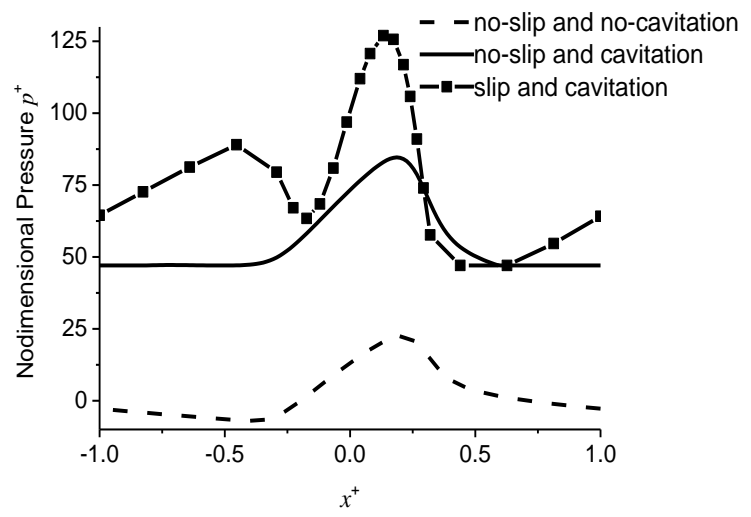

Fig. 5: Pressure distribution on the upper wall under different slip conditions with $w^{+}=0.3$ and $d^{+}=0.5$

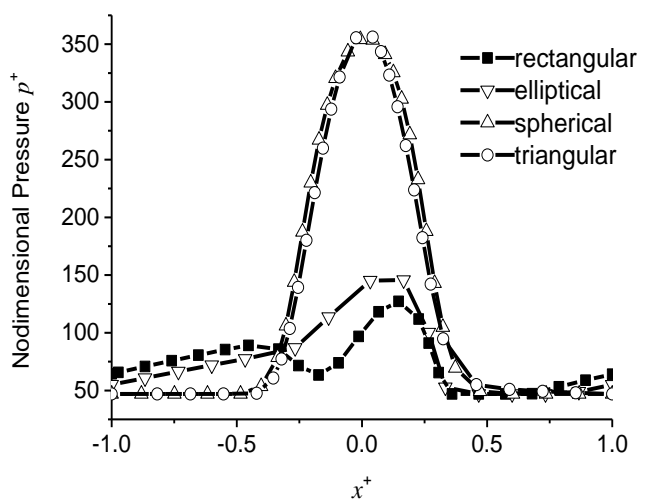

Fig. 6: Pressure distribution on the upper wall when micro textures with various geometric features are introduced,

$$
w^{+}=0.3, \quad d^{+}=0.5
$$

\subsection{Effect of the geometry size of textures on hydrodynamic lubrication}

A spherical cross shape model, as shown in Figure 1 (b) is used to analyze the effect of the micro texture on the fluid lubrication properties.

\section{1) Effect of the texture width on hydrodynamic lubrication}

In order to analyze the influence of the micro texture width to the lubrication properties, the dimensionless depth $d^{+}=0.5$ is fixed and the dimensionless width $w^{+}=0.15,0.2,0.25,0.3,0.35,0.4,0.45,0.5$ are adopted respectively. The influence of the micro texture width on the fluid lubrication performance is shown in figure 7.

It can be seen from figure 7 that the friction force decrease due to the wall slip and the bearing capacity of lubrication film increases with the width of micro texture. When $w^{+}<0.2$, the friction decreases with the increase of the width of the micro texture and the friction comes to the minimum value at $w^{+}=0.2$ when 
complete slip condition is taken. When no slip occurs, the magnitude of friction decreases after $w^{+}>0.2$ and is closer to each other in two cases when $w^{+}=0.5$.

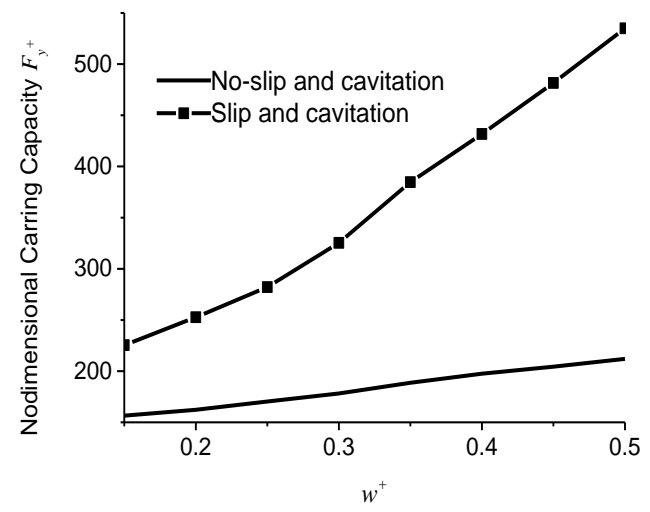

(a) Capacity

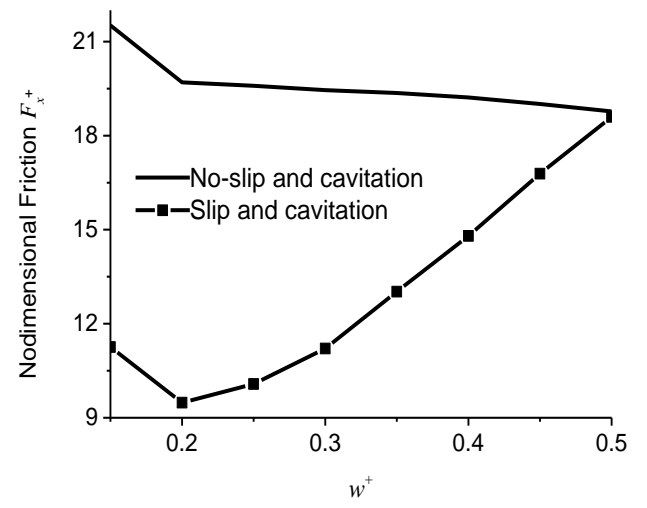

(b) Friction

Fig. 7: Influence of the width of micro texture on the lubrication properties

\section{2) The influence of the depth of micro texture}

In order to analyze the influence of the micro texture depth on the lubrication properties, the dimensionless width $w^{+}=0.3$ was fixed and various dimensionless micro texture depth involving $d^{+}=0.5,0.75$, $1,1.25,1.5$ were adopted.

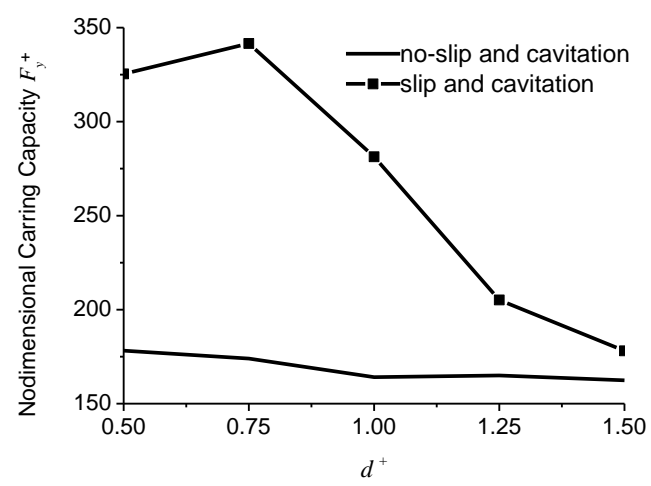

(a) Carrying capacity

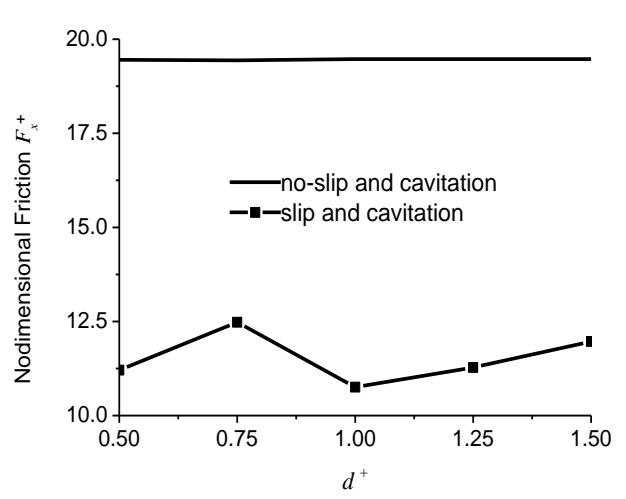

(b) Friction

Fig. 8: Hydrodynamic lubrication versus texture depth for different conditions at $w^{+}=0.3$

It can be seen from figure 8 that for the case of slip and cavitation conditions, both the carrying capacity and friction increase with the increase of texture depth when nodimensional depth is less than 0.75 . The maximum carrying capacity is obtained at $d^{+}=0.75$ and then diminishes considerably. The friction force obtains the minimum value at $d^{+}=1.0$. When cavitation is considered only, both carrying capacity and friction change a little with the increase of texture depth.

From the numerical results, it can be seen that the cavitation boundary condition have an important influence on the pressure distribution in the fluid field and then impose a significant effect on the lubrication properties especially on the load capacity. Of course, this is just theoretical calculation results. In subsequent work, it is necessary to verify experimentally whether there exist significant cavitation and under what conditions cavitation will occur.

\section{Conclusions}

The wall slip influence on lubricating properties considering cavitation effect has been numerically studied using CFD software and the following conclusions were drawn:

1) The wall slip reduces the friction force of the lubrication film and the cavitation boundary condition have a significant influence on the simulation results of carrying capacity; Then it is important for 
subsequent experimental work to verify whether there exist significant cavitation and under what conditions cavitation will occur.

2) Compared with texture with cross section of triangle, rectangle and ellipse, the spherical texture facilitates carrying capacity improvement. There exists the optimal texture geometry size that facilitates lubrication property optimization. Under the parameters used in the work, the friction coefficient is the smallest when the dimensionless width of the micro texture is 0.35 and the friction coefficient will increase when the dimensionless texture depth is more than 1.0.

\section{Acknowledgements}

This research was financially supported by the National Science Foundation of China (51775431, 51675422, 51375381).

\section{References}

[1] C. Wu, G. Ma and P. Zhou. A review of the study on the boundary slip problems of fluid flow. Advances in Mechanics, 2008, 38(3): 265-282.

[2] T. Sochi. Slip at fluid-solid interface. Polymer Reviews, 2011, 51(4):309-340.

[3] C. Lee, C. Choi and C. Kim. Structured surfaces for a giant liquid slip. Physical Review Letters, 2008, 101(6):064501-4.

[4] H. Spikes. The half-wetted bearing. Part1:extended Reynolds equation. Proceedings of the Institution of Mechanical Engineers, Part J: Journal of Engineering Tribology, 2003, 217(1): 1-14.

[5] H. Zhang, P. Zhang, H. Meng, et al. Boundary slip design of high speed water lubricated journal bearing. Journal of Xi'an Jiaotong University, 2013, 47(9): 72-90.

[6] G. Ma, C. Wu and P. Zhou. Multi-linearity algorithm for wall slip in two-dimensional gap flow. International Journal for Numerical Methods in Engineering, 2007, 69(12):2469-2484.

[7] G. Ma. Boundary Slip and Hydrodynamics of Fluid Flow in a Micro/Nano-Gap. Doctoral Thesis, Dalian University of Technology, 2007.

[8] S. Wang, K. Liu, X. Yao, et al. Bioinspired surfaces with superwettability: new insight on theory, design and application. Chemical Reviews, 2015, 115(16): 8230-8293.

[9] Y. Yan and N. Gao. Barthlott W. Mimicking natural superhydrophobic surfaces and grasping the wetting process: A review on recent progress in preparing superhydrophobic surfaces. Advances in Colloid \& Interface Science, 2011, 169(2):80.

[10] Y. Zhang, Y. Chen, L. Shi, et al. Recent progress of double-structural and functional materials with special wettability. Journal of Materials Chemistry, 2011, 22(3):799-815.

[11] I. Etsion, G. Halperin, V. Brizmer, et al. Experimental investigation of laser surface textured parallel thrust bearings. Tribology Letters, 2004, 17(2): 295-300.

[12] X. Wang, K. Koji, A. Koshi, et al. The effect of laser texturing of SiC surface on the critical load for the transition of water lubrication mode from hydrodynamic to mixed. Tribology International, 2001, 34:703-711.

[13] D. Hamilton, J. Walowit, C. Allen. A Theory of Lubrication by Microirregularities. Journal of Fluids Engineering, 1966, 88(1):177-185.

[14] M. Tauviqirraham, J. Muchammad, D. Schipper. Numerical study of the load-carrying capacity of lubricated parallel sliding textured surfaces including wall slip. Tribology Transactions, 2014, 57( 1):134-145.

[15] X. Lu, Q. Wang, J. Xiao, Z. Yang. CFD Analysis on the effect of cavitation of textured surface on hydrodynamic lubrication. Lubrication Engineering, 2016, 41(5):70-75.

[16] G. Schnerr, J. Sauer. Physical and Numerical Modeling of Unsteady Cavitation Dynamics. In: Fourth International Conference on Multiphase Flow, New Orleans, USA, 2001, pp. 1-8.

L. Wang L, C. Lu, J. Ma, et al. Study on two-dimensional wall slip of sleeve bearing. Journal of mechanical engineering, 2012, 48(7):105-112. 